\title{
Structural Variations in Hybrid All-Nanoparticle Gibbsite Nanoplatelet/Cellulose Nanocrystal Multilayered Films
}

Clélia Martin, ${ }^{+,}{ }^{\ddagger} \S$ Robert Barker, ${ }^{\S, 1}$ Erik B. Watkins, ${ }^{\S, 2}$ Frédéric Dubreuil, ${ }^{+,}$Emily D. Cranston, ${ }^{\prime \prime}$

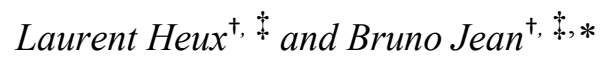

${ }^{\dagger}$ Univ. Grenoble Alpes, Centre de Recherches sur les Macromolécules Végétales (CERMAV), F-38000 Grenoble, France

\$CNRS, CERMAV, F-38000 Grenoble, France

$\S$ Institut Laue-Langevin, Grenoble, France

" Department of Chemical Engineering, McMaster University, Hamilton, Ontario L8S 4L7, Canada

\begin{abstract}
Cellulose nanocrystals (CNCs) are promising bio-sourced building blocks for the production of high performance materials. In the last ten years, $\mathrm{CNCs}$ have been used in conjunction with polymers for the design of multilayered thin films via the layer-by-layer assembly technique. Herein, polymer chains have been replaced with positively charged inorganic gibbsite nanoplatelets $(\mathrm{GN})$ to form hybrid "nanoparticle-only" composite films. A combination of atomic force microscopy and neutron reflectivity experiments was exploited to investigate the growth and structure of the films. Data show that the growth and density of GN/CNC films can be tuned over a wide range during preparation by varying the ionic strength in the CNC suspension and the film drying protocol. Specifically, thin and dense multilayered films or very thick, more porous mixed slabs, as well as intermediate internal structures could be obtained in a predictable manner. The influence of key physicochemical parameters on the multilayer film build up was elucidated and the film architecture was linked to the dominating interaction forces between components. The degree of structural control over these hybrid nanoparticle-only films is much higher than that reported for $\mathrm{CNC}$ /polymer films, which offers new properties and potential applications as separation membranes or flame retardant coatings.
\end{abstract}




\section{Introduction}

Scientific and industrial communities are constantly looking for materials with improved performances and new functionalities. In this context, the versatile and robust layerby-layer (LbL) assembly concept, first described in 1966 by Iler $^{1}$ and widely developed over the last 20 years has led to highly interesting achievements in soft matter and materials science. ${ }^{2-}$ ${ }^{4}$ By sequential adsorption of two different components with mutually attractive interactions, the LbL method allows for the design of multilayered thin films with precise control of the nano-scale architecture. First developed for inorganic colloidal particles and later for polyelectrolytes, LbL has been applied on a broad variety of substrates with components ranging from organic colloids to carbon nanotubes, proteins, polysaccharides, biomacromolecules and even whole microorganisms. These developments are well summarized in numerous review papers ${ }^{5-8}$ and in a comprehensive book co-written by many experts in the field. ${ }^{9}$

Among the suitable components that can be used to build LbL-based structures with enhanced physical properties, cellulose nanocrystals (CNCs) stand out as promising candidates. ${ }^{10-12}$ Indeed, CNCs, which are now commercially available in large quantities, display outstanding mechanical properties such as an elastic modulus as high as $120-150 \mathrm{GPa}^{13}$ and a strength up to $6 \mathrm{GPa},{ }^{14}$ together with a high aspect ratio and a relatively low density. In addition, CNCs are biodegradable and biocompatible, and as such appear today as perfect building blocks for the design of eco-friendly multilayer products with tunable internal structure.

CNCs have already been used in conjunction with various polymers in LbL structures as shown in a recent review. ${ }^{15}$ Originally, CNCs were combined with synthetic polycations such as poly(diallyldimethylammonium chloride) (PDDA), ${ }^{16}$ polyethyleneimine (PEI) ${ }^{17}$ or polyallylamine hydrochloride $(\mathrm{PAH})^{18-21}$ based on electrostatic interactions, whereby different film architecture and build up were observed depending on the type of CNCs, polymer, and deposition method/protocol used. ${ }^{22}$ Specifically, the ionic strength of the polycation solution, the film drying process, the CNC concentration and the dipping time have been shown to be key parameters that influence the average thickness increment per deposited bilayer. More recently, completely "green" CNC multilayers have been built, also based on electrostatic 
interactions, using natural polycations like chitosan ${ }^{23}$ and collagen ${ }^{24}$ or based on specific nonelectrostatic interactions, such as the case with CNCs and xyloglucan, a neutral polysaccharide. ${ }^{25-29}$

In recent developments, the mixture of cellulose nanofibrils (another type of nanocellulose distinct from CNCs) with clay nano platelets has proven extremely promising. Indeed, these mixtures yielded multi-layered structures that present outstanding mechanical strength and toughness and in some cases, good transparency and excellent gas barrier properties. ${ }^{30-33}$ Additionally, multilayered products with mechanical properties approaching those of nacre have been prepared using LbL with PDDA and anionic montmorillonite clay. ${ }^{34}$ Inspired by the success of such a simple process and following our desire to develop new bioinspired cellulose-based structurally-tunable materials, this work explores the assembly of thin films consisting of CNCs and inorganic nanoplatelets.

Herein we describe the preparation of LbL nano-structured films consisting of alternating layers of CNCs and nanoplatelets of gibbsite. The latter are indeed very interesting model colloids to be associated with CNCs since they are in the form of individualized hexagonal platelets with positive surface charges and exhibit well-defined dimensions, colloidal stability and liquid-crystal phases self-organization behavior. ${ }^{35-37}$ To the best of our knowledge, LbL films incorporating CNCs have either been combined only with polymers (either charged or neutral, as described above) or with the help of polyelectrolytes when other nanoparticles like carbon nanotubes were also present, ${ }^{38-39}$ making this the first demonstration of polymer-free CNC-inorganic nanoparticle hybrid layered films. Atomic force microscopy (AFM) was used to study the surface topography and measure the total film thickness and neutron reflectivity (NR) at the solid/air interface was used to extract the particle volume fraction along the normal to the film, i.e. the film's porosity, as well as the roughness. We have identified the key physicochemical parameters that influence multilayer growth. Overall, a comprehensive picture of the film architecture for "nanoparticle-only" composites has emerged along with a better understanding of the dominant interaction forces during LbL film assembly.

\section{Experimental section}

\section{Polymers and chemicals}

Polyethyleneimine (PEI. $M_{\mathrm{W}} \sim 25000$ ), poly(allylamine hydrochloride) (PAH. $M_{\mathrm{W}} \sim 70000$ ), poly(sodium 4-styrene sulfonate) (PSS. $M_{\mathrm{W}} \sim 70000$ ), aluminium isopropoxide (M=204.25 
$\mathrm{g} / \mathrm{mol})$ and aluminium secbutoxide $(\mathrm{M}=246.33 \mathrm{~g} / \mathrm{mol})$ were purchased from Sigma-Aldrich and used as received. Cotton linters were provided by Buckeye Cellulose Corporation and used as the cellulose source for CNCs without any further purification. Deionized water was systematically used for the preparation of suspensions.

\section{Cellulose nanocrystals (CNCs)}

CNCs were obtained from the hydrolysis of cotton linters with small modifications to the method described by Revol et al. ${ }^{40}$ Briefly, the cellulose was treated with 65 wt. \% sulfuric acid for 30 minutes at $63{ }^{\circ} \mathrm{C}$. The suspensions were washed by centrifugation, dialyzed against distilled water, and sonicated for 4 min with a Branson model 450 sonifier at 30\% intensity. After these treatments, the suspensions were filtered through $5 \mu \mathrm{m}$ and then $1 \mu \mathrm{m}$ cellulose nitrate membranes (Sartorius) and the resulting 2.3 wt. \% suspension was stored at $4^{\circ} \mathrm{C}$. To avoid any bacterial contamination, some chloroform was added to the suspension. CNC suspensions were concentrated by ultrafiltration using $10000 \mathrm{kDa}$ ultrafiltration discs (Millipore) to $3.6 \mathrm{wt}$ \%, which was used for multilayer film build up. After concentration, the $\mathrm{pH}$ of the suspension was 2.3 , i.e., the CNCs were used in the acidic form. Transmission electron microscopy (Philips CM200 'Cryo' electron microscope operating at $80 \mathrm{kV}$ ) and AFM were used to extract the average dimensions of the particles, $150 \mathrm{~nm} \times 22 \mathrm{~nm} \times 6 \mathrm{~nm}$, similar to those previously reported. ${ }^{41-42}$ Conductometric titration and elemental analysis gave sulfur content values equal to $0.67 \%$ and $0.71 \%$, respectively. Combining these values with the size of the particles allowed us to estimate the average surface charge density as $0.48 \mathrm{e}^{-} / \mathrm{nm}^{2}$. A zeta potential of $-46 \pm 5 \mathrm{mV}$ was determined with a NanoZS instrument from Malvern on a $0.1 \mathrm{wt}$. $\%$ CNC suspension.

\section{Gibbsite nanoplatelets (GN)}

Gibbsite nanoplatelets (GN) were obtained by hydrothermal treatment of aluminium isopropoxyde $(0.08 \mathrm{M})$ and aluminium secbutoxide $(0.08 \mathrm{M})$ in hydrochloride acid $(0.09 \mathrm{M}){ }^{36}$ After dissolution for 10 days, the suspension was heated for 72 hours at $85{ }^{\circ} \mathrm{C}$ in an oven. The suspension was then dialyzed against distilled water until neutrality and concentrated by ultrafiltration to reach $5 \mathrm{wt}$. \%. The $\mathrm{pH}$ of the GN suspension was set at 5.3. The diameter of the platelets was obtained from transmission electron microscopy observations. About 100 particles were analyzed using the Image J software and an average diameter of $105 \mathrm{~nm}$ with a standard deviation of $41 \mathrm{~nm}$ was found. To determine the thickness of GN, a drop of an extremely dilute GN suspension was first deposited on a mica slide and then quickly removed 
in order to adsorb single and isolated particles. The thickness was then determined by AFM height analysis and found to be ca. $4 \mathrm{~nm}$. According to the literature, the surface charge density of the gibbsite colloids is $5+\mathrm{e} / \mathrm{nm}^{2} .{ }^{37}$ The zeta potential of a $0.1 \mathrm{wt}$. $\%$ GN suspension was +47 $\pm 5 \mathrm{mV}$.

\section{Layer-by-Layer (LbL) assembly}

Multilayered films were assembled on polished silicon $<001>$ wafers (Wafer World Inc.). The solid substrates were cleaned with a Piranha $\mathrm{H}_{2} \mathrm{SO}_{4} / \mathrm{H}_{2} \mathrm{O}_{2}$ mixture $(70: 30 \mathrm{v} / \mathrm{v})$ at room temperature for $30 \mathrm{~min}$, followed by intensive rinsing with water. The Piranha cleaning generates negative charges at the silicon surface. Prior to the deposition of the first GN layer, a polyelectrolyte multilayer "primer" composed of PEI/PSS/PAH/PSS was deposited on the silicon substrate. Such a procedure aims at obtaining a highly uniform and reproducible surface charge density that is independent of the exact substrate surface chemistry. Thin films were then assembled using LbL with dipping; the negatively charged substrate was first immersed in the positively-charged GN suspension for a determined dipping time, $t$, the substrate was rinsed for $5 \mathrm{~min}$ with distilled water and then the substrate was immersed in the negatively charged $\mathrm{CNC}$ suspension for an identical time, $t$, and then rinsed for $5 \mathrm{~min}$. The rinsing steps are required to remove loosely bound nanoparticles and ensure that only strongly interacting particles are adsorbed. This cycle was repeated to reach the desired number of deposited bilayers, $n$. The dipping time $t$ was varied between $10 \mathrm{~s}$ and $15 \mathrm{~min}$. Additionally, to obtain the final dried films, two different drying protocols were used:

In protocol I (intermediate drying), samples were dried using gentle blowing of air after each dipping + rinsing step. i.e., after each layer deposited.

In protocol F (final drying only), intermediate drying steps were omitted and samples were built in aqueous environment and dried only one time after the last layer had been deposited.

Accordingly, the notation $(\mathrm{GN} / \mathrm{CNC})_{n . \mathrm{I}}$ or $(\mathrm{GN} / \mathrm{CNC})_{n . \mathrm{F}}$ designates a multilayered film containing $n$ number of deposited GN/CNC bilayers, dried using protocol I or F, respectively. When $n$ is equal to a half integer, then the film is terminated by a GN layer. In some cases, $x$ $\mathrm{mM}$ of $\mathrm{NaCl}$ was added to the $\mathrm{CNC}$ suspension which is then denoted $(\mathrm{GN} / \mathrm{CNC} x \mathrm{mM} \mathrm{NaCl})_{n . \mathrm{I}}$ or F. The polyelectrolyte primer layer is omitted in the notation for clarity.

\section{Transmission electron microscopy}


Drops of ca. $0.001 \mathrm{wt} \%$ GN or CNC suspensions were deposited onto glow-discharged carboncoated TEM grids. After 2 min, the liquid in excess was absorbed with filter paper and, for CNCs only, prior to drying, a drop of 2\% uranyl acetate was deposited on the specimen. After $2 \mathrm{~min}$, the stain in excess was blotted and the remaining thin liquid film was allowed to dry. The specimens were observed using a Philips CM200 electron microscope operating at $80 \mathrm{kV}$. The images were recorded on Kodak SO163 films.

\section{AFM measurements}

AFM experiments were performed using a PicoPlus (Molecular Imaging) commercial instrument at the PCI-ICMG platform. Topography height images were obtained in tapping mode with silicon tips and cantilevers coated with aluminium $(48 \mathrm{~N} / \mathrm{m}, 190 \mathrm{kHz}-$ Vista Probes, Nanoscience Instruments). Data treatment and presentation of the images was carried out using the Gwyddion Software package (http://gwyddion.net). Thickness was derived from scratchheight analysis (films were scratched using tweezers) and rms roughness was evaluated from 5 $\mu \mathrm{m} \times 5 \mu \mathrm{m}$ images. Thickness and roughness values correspond to the average of measurements on two different samples at 3 different positions for each sample. The corresponding standard deviation was calculated from these 6 values.

\section{Neutron reflectivity}

Specular neutron reflectivity was used for the structural characterization of thin films in the direction perpendicular to the reflecting surface. Neutrons are sensitive to the atomic nuclei and the interaction between neutrons and matter is defined by the scattering length density (SLD) also noted $\rho$. The scattering length density, $\rho_{\text {film }}$, of a (GN/CNC) multilayered film is equal to: $\rho_{\mathrm{film}}=\rho_{\mathrm{GN}} . \phi_{\mathrm{GN}}+\rho_{\mathrm{CNC}} . \phi_{\mathrm{CNC}}+\rho_{\mathrm{air}} . \phi_{\mathrm{air}}($ Equation 1$)$

Where $\phi_{\mathrm{GN}}, \phi_{\mathrm{CNC}}$ and $\phi_{\mathrm{air}}$ and $\rho_{\mathrm{GN}}, \rho_{\mathrm{CNC}}$ and $\rho_{\mathrm{air}}$ are the particle volume fractions and scattering length densities of GN, CNC and air, respectively. Since the scattering length densities of air, CNCs and GN are respectively equal to $0,1.9$ and $1.810^{-6} \AA^{-2}$, Equation 1 can crudely be simplified to:

$\phi_{\mathrm{GN}}+\phi_{\mathrm{CNC}}=\rho_{\mathrm{film}}\left(\right.$ in $\left.\AA^{-2}\right) / 1.85 \times 10^{-6}($ Equation 2$)$

This simplification based on the very small difference in SLD between the GNs and the CNCs 
reduces the number of fitted parameters in the data while still enabling access to the total particle volume fraction and hence the porosity $\left(\phi_{\text {air }}\right)$ in a $(\mathrm{GN} / \mathrm{CNC})$ film from $\phi_{\mathrm{air}}=1-\left(\phi_{\mathrm{GN}}+\phi_{\mathrm{CNC}}\right) \quad($ Equation 3$)$

Specular neutron reflectivity experiments were carried out on the time-of-flight reflectometers FIGARO and D17 at the Institut Laue-Langevin (ILL), Grenoble, France. ${ }^{43}$ The reflectivity profiles of films $(\mathrm{GN} / \mathrm{CNC})_{n}$. I with $n=2,3,4$ and 7 and films (GN/CNC) ${ }_{n}$. F with $n=2,4$ and 7 were measured on FIGARO in the time of flight mode for two different incident angles $\left(0.624^{\circ}\right.$ and $\left.3^{\circ}\right)$ to cover a q-range ranging from 0.008 to $0.3 \AA^{-1}$ with a $7 \% \Delta \mathrm{Q} / \mathrm{Q}$ resolution. The thickest samples (i.e., for $n=7$ ) were measured only at $0.624^{\circ}$ at a higher $\Delta \mathrm{Q} / \mathrm{Q}$ resolution $(2 \%)$.

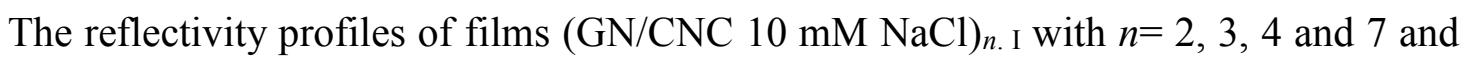
films $(\mathrm{GN} / \mathrm{CNC} 10 \mathrm{mM} \mathrm{NaCl})_{n}$. F with $n=2,4$ and 7 were measured on D17 in the time of flight mode for two different incident angles $\left(0.5^{\circ}\right.$ and $\left.1.5^{\circ}\right)$ to cover a q-range ranging from 0.008 to $0.3 \AA^{-1}$ with a $2 \% \Delta \mathrm{Q} / \mathrm{Q}$ resolution. All spectra recorded at the solid/air interface, i.e., dry films, were fitted with the Motofit software (http://motofit.sourceforge.net) taking into account the per point angular resolution of the reflectometer. A box model was used which involved dividing the thin film into a series of layers, each layer being characterized by a finite thickness, scattering length density and interfacial roughness with the neighboring layer.

To study never-dried (GN/CNC $10 \mathrm{mM} \mathrm{NaCl})_{n . \mathrm{N}}$ with $n=2$ and 4 , the reflectivity at the solid/liquid interface was measured on FIGARO using a cell designed at the ILL. Samples were measured at two angles $\left(0.622^{\circ}\right.$ and $\left.1.98^{\circ}\right)$ with a $0.82 \%$ resolution. The resulting spectra were fitted using the RasCAL fitting package (https://sourceforge.net/projects/rscl/).

\section{Contact angle measurements}

The contact angle measurements were performed using an OCA 35 apparatus from Dataphysics - Apollo Instruments. Images of a sessile drop at the points of intersection between the drop contour and the projection of the surface were recorded by a camera and a drop shape analysis was performed by the software to give the contact angle. The drop volume was fixed at $5 \mu \mathrm{L}$. For each point, 5 measurements were done at different positions on the film surface and an average value with the corresponding standard deviation is reported. 


\section{Zeta potential}

The zeta potential of CNC and GN particles in suspension was measured by electrophoresis coupled with laser Doppler velocimetry using a Malvern NanoZS instrument. All samples were measured at $0.01 \mathrm{M} \mathrm{NaCl}$. Data were averaged over 3 measurements.

\section{Surface Zeta potential}

Zeta potential measurements were performed with an electrokinetic analyzer (SurPASS from Anton Paar GmbH, Graz, Austria). The measurements were performed using $1 \mathrm{mM} \mathrm{KCl}$ in DI water as the electrolyte. Two multilayered film-covered substrates of $20 \mathrm{~mm} \times 10 \mathrm{~mm}$ were used for the measurement and mounted using the adjustable-gap cell. The zeta potential was calculated from the streaming potential and streaming current measurements based on the Smoluchowski equations. 


\section{Results}

\section{Building hybrid films}

As proof of principle that GNs and CNCs interact favorably and lead to multilayer film build up, GN/CNC films were first assembled using drying protocol I (with intermediate drying) and no additional salt, with a dipping time set to $15 \mathrm{~min}$. As in all subsequent experiments, the concentration and $\mathrm{pH}$ of the GN and CNC suspensions were fixed to $5.0 \mathrm{wt}$. $\% / \mathrm{pH} 5.3$ and 3.6 wt. \%/pH 3.0, respectively. Under these conditions, both the faces and the edges of GNs are positively charged, as shown by Wierenga et al., ${ }^{37}$ and the surface sulfate half esters of the CNCs are fully dissociated, ${ }^{44}$ making the particles negatively charged. Visual inspection of the films was carried out after each bilayer deposition. Additionally, photographs were taken under the exact same illumination and film-camera lens angular conditions and the resulting series is shown in Figure 1.

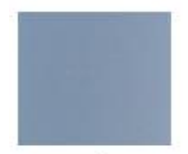

1

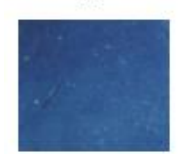

5

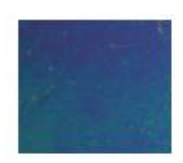

9

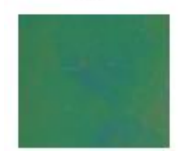

13

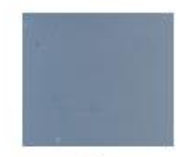

2

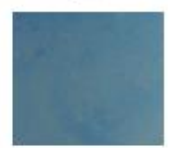

6

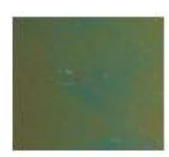

10

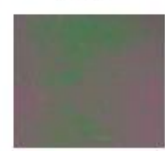

14

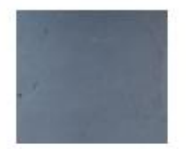

3

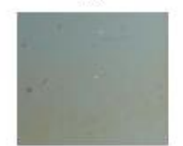

7

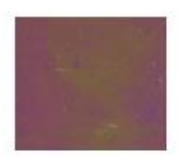

11

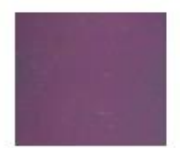

15

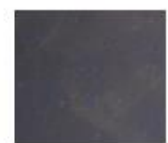

4

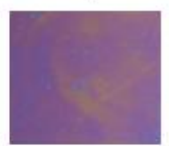

8

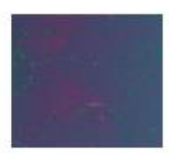

12

Figure 1. Interference colors displayed by $(\mathrm{GN} / \mathrm{CNC})_{n, \mathrm{I}}$ films as a function of the number of deposited bilayers, $n$ (indicated below each film). Images were recorded under the same illumination and angular conditions. Each square represents a film area of $1.4 \mathrm{~cm} \times 1.4 \mathrm{~cm}$.

The films display intense angle-dependent colors that are related to thin film thickness. 
Colors arise from the interference between the light reflected from the film/air interface and from the film/Si substrate interface and these colors were found to be fairly homogeneous over the whole film surface. A pronounced color change was detected after each newly adsorbed bilayer, as shown in Figure 1, which supports the growth of the film with increasing $n$. For $n<5$, grey tones are observed since the films are not thick enough to yield colors (film thickness is discussed quantitatively in a later section). Such interference colors have already been reported for polymer/CNC multilayered films. ${ }^{15}$ These observations imply that the all-nanoparticle GN/CNC films can be sequentially assembled and are uniform and smooth enough in the planar direction to allow for the separation of the different colors from the white light spectrum.

AFM topography images of samples $(\mathrm{GN} / \mathrm{CNC})_{n . \mathrm{I}}$ for $n=0.5,1$ and 2.5 are shown in Figure 2. Figure $2 \mathrm{a}(n=0.5)$ corresponds to the first layer of gibbsite nanoplatelets deposited on top of the polyelectrolyte primer. The GNs lay flat on top of the low-roughness polymer layer and no pronounced tilt is observed even in places where platelets overlap. A strikingly high particle density is observed, which supports strong electrostatic interactions between the GN layer and the PSS-terminated polyelectrolyte primer.
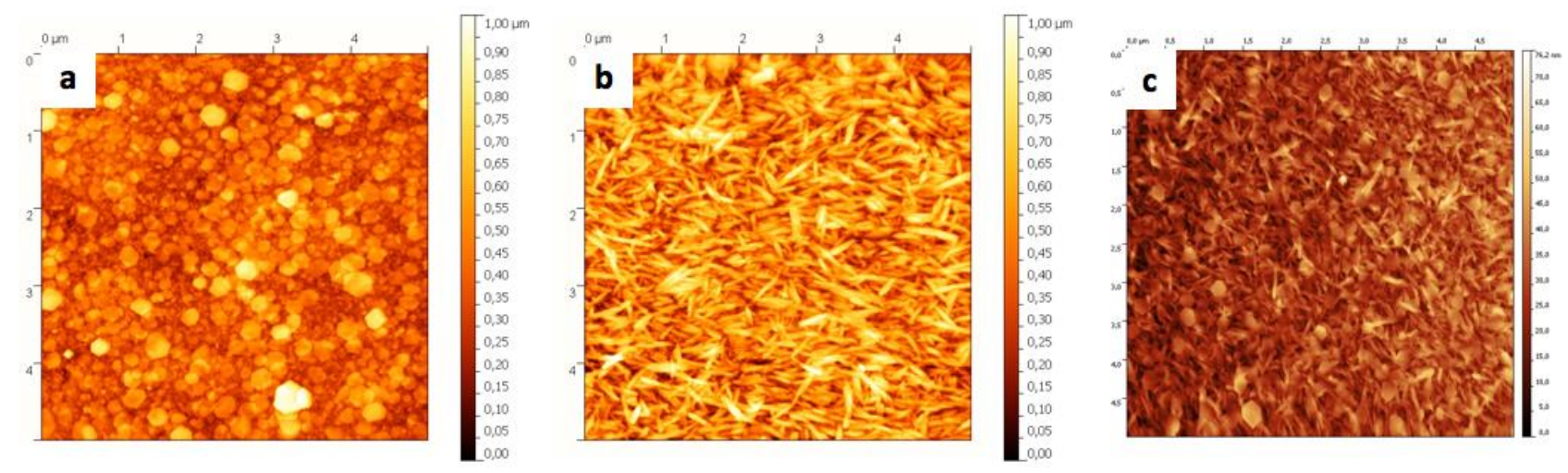

Figure 2. $5 \mu \mathrm{m} \times 5 \mu \mathrm{m}$ AFM topography images of films (GN/CNC) ${ }_{n, \mathrm{I}}$ for $n=0.5$ (a), 1 (b) and 2.5 (c).

Figure $2 \mathrm{~b}(n=1)$ corresponds to the next deposition step, i.e. after adsorbing CNCs on top of the first GN layer. Again, a very high nanoparticle density is observed. Interestingly, CNCs cover the platelets so densely that no underlying platelets can be distinguished, suggesting strong interactions between the GNs and the CNCs. No preferential orientation of the CNCs is detected and when looking carefully at the image, it can be noted that the CNC layer consists of more than a monolayer. Figure $2 \mathrm{c}(n=2.5)$ shows that GNs were undeniably adsorbed on the (GN/CNC)2,I surface but compared to Figure 2a, the platelets are more tilted. This is probably due to the fact that GNs are no longer lying on a smooth polyelectrolyte surface 
but are now on a rougher CNC layer. The adsorption density of GN for $n=2.5$ cannot be easily estimated since some platelets lay flat and others are tilted.

\section{Tuning the growth of the films}

The aforementioned results demonstrate our ability to build hybrid all-nanoparticle GN/CNC multilayered films. In the next step, we investigated the influence of two parameters on the growth and structure of the films, namely the ionic strength of the $\mathrm{CNC}$ suspension ( 0 or $10 \mathrm{mM}$ added $\mathrm{NaCl}$ ) and the drying protocol, namely with protocol I (intermediate drying), samples were dried using gentle blowing of air after each dipping + rinsing step. i.e., after each layer deposited, whereas with protocol F (final drying only), intermediate drying steps were omitted and samples were built in aqueous environment and dried only one time after the last layer had been deposited.

The combination of these two parameters gives four different systems, which were first investigated by AFM to obtain the film thickness as a function of the number of deposited bilayers for $n$ up to 15. Film thickness results are summarized in Figure 3.

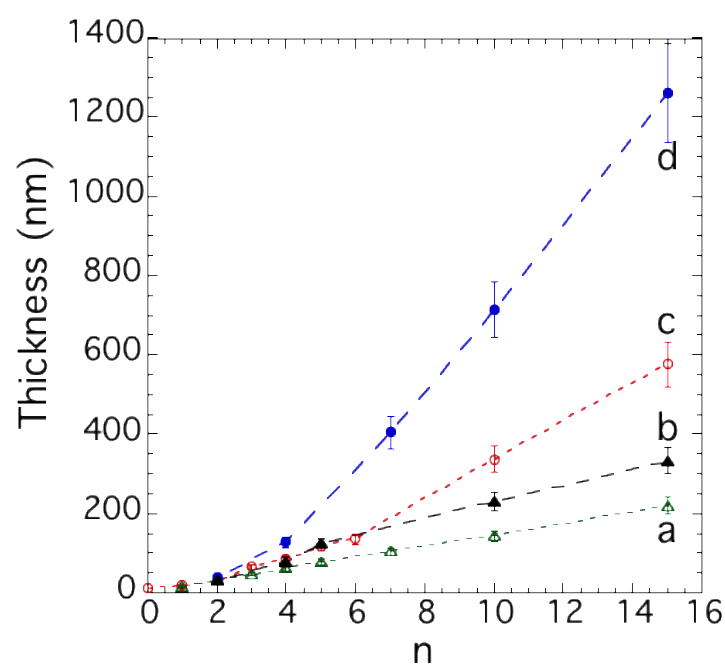

Figure 3. Evolution of film thickness with the number of deposited bilayers, $n$, for (GN/CNC $10 \mathrm{mM} \mathrm{NaCl})_{n, \mathrm{I}}(\mathrm{a}),(\mathrm{GN} / \mathrm{CNC} 10 \mathrm{mM} \mathrm{NaCl})_{n, \mathrm{~F}}(\mathrm{~b}),(\mathrm{GN} / \mathrm{CNC})_{n, \mathrm{I}}(\mathrm{c})$ and $(\mathrm{GN} / \mathrm{CNC})_{n, \mathrm{~F}}(\mathrm{~d})$ multilayered films.

A thickness increase is observed for each of the four conditions probed, showing successful film growth in all cases. However, four significantly different film growth profiles are obtained showing a strong influence of both the ionic strength and the drying protocol. In the presence of $10 \mathrm{mM} \mathrm{NaCl}$ in the $\mathrm{CNC}$ suspension (Figure 3, lines a and b), linear film growth 
was observed over the whole $n$ range and was independent of the drying protocol. Average thickness increments per bilayer of 14 and $23 \mathrm{~nm}$ were found for films (GN/CNC $10 \mathrm{mM}$ $\mathrm{NaCl})_{n, \mathrm{I}}$ and $(\mathrm{GN} / \mathrm{CNC} 10 \mathrm{mM} \mathrm{NaCl})_{n, \mathrm{~F}}$, respectively. These values are on the same order of magnitude as those obtained for polymer/CNC films. ${ }^{15}$

Conversely, without added salt (Figure 3, lines c and d) a much faster growth was observed with increasing $n$. Under these conditions, the film build up can be divided into two regimes. For $n<4$, the average thickness increment per bilayer is low and non-linear. For $n \geq 5$, a steady-state regime characterized by a steep linear growth was observed, with thickness increments per bilayer of 48 and $100 \mathrm{~nm}$ for $(\mathrm{GN} / \mathrm{CNC})_{n, \mathrm{I}}$ and $(\mathrm{GN} / \mathrm{CNC})_{n, \mathrm{~F}}$, respectively. Such values are very high and allow film thicknesses of 580 and $1260 \mathrm{~nm}$ to be reached within 15 deposited bilayers for $(\mathrm{GN} / \mathrm{CNC})_{n, \mathrm{I}}$ and $(\mathrm{GN} / \mathrm{CNC})_{n, \mathrm{~F}}$, respectively. Such a rapid thickness increase had never been reported for CNC-based LbL films. ${ }^{15}$

For a given drying protocol and for $n \geq 5$, the increase in ionic strength results in about 2 to 4 times thinner films than in the absence of added salt. Additionally, as shown in Supporting Information Figure $\mathrm{S} 1,(\mathrm{GN} / \mathrm{CNC} x \mathrm{mM} \mathrm{NaCl})_{n, \mathrm{I}}$ films exhibit very similar thickness profiles for $x=1,5$ and $10 \mathrm{mM}$ salt, in the $5 \leq n \leq 15$ range. This shows that the addition of monovalent salt to the CNC suspension, even with concentrations as low as $1 \mathrm{mM}$, has a substantial effect on the film growth. Further increasing the ionic strength leads to similar growth behavior in the $\mathrm{NaCl}$ concentration range probed.

Irrespective of ionic strength conditions, Figure 3 clearly demonstrates that the absence of intermediate drying, i.e., protocol $\mathrm{F}$, results in films that are approximately two times thicker compared to the protocol I case. Films assembled following protocol $\mathrm{F}$ also displayed structural colors but unlike the ones made with protocol I (shown in Figure 1), the colors are inhomogeneous (results not shown). A very pronounced effect of the drying protocol is therefore observed.

The build up and structure of LbL films assembled using the dipping technique can be sensitive to the dipping time; rather long times, on the order of 10 minutes, may be required to reach thermodynamic equilibrium. ${ }^{45}$ The influence of the dipping time on the construction of $(\mathrm{GN} / \mathrm{CNC} 10 \mathrm{mM} \mathrm{NaCl})_{n, \mathrm{I}}$, and $(\mathrm{GN} / \mathrm{CNC} 10 \mathrm{mM} \mathrm{NaCl})_{n, \mathrm{~F}}$ films was thus investigated and results are shown in Supporting Information Figures S2 and S3. Remarkably, when intermediate drying was applied (protocol I, Figure S2), similar linear film growth was observed for $\mathrm{t}=10 \mathrm{~s}, 1 \mathrm{~min}, 5 \mathrm{~min}$ and $15 \mathrm{~min}$ in the $2 \leq n \leq 15$ range. This is significant because a reduction of the dipping time down to $10 \mathrm{~s}$ allows for rapid production of thick films and suppresses one of the major drawback of the dipping method (at the expense of intermediate drying steps). 
On the other hand, for films assembled with protocol $\mathrm{F}$ for two different dipping times, 15 min and $10 \mathrm{~s} \mathrm{(Figure} \mathrm{S3),} \mathrm{radically} \mathrm{different} \mathrm{thicknesses} \mathrm{were} \mathrm{obtained.} \mathrm{Dipping} \mathrm{for} \mathrm{only} 10$ $\mathrm{s}$ leads to much thinner films (about half as thick as $15 \mathrm{~min}$ dipped films). The thickness increment per bilayer decreases from $21 \mathrm{~nm} /$ bilayer for $15 \mathrm{~min}$ dipping to about $10 \mathrm{~nm} / \mathrm{bilayer}$ for $10 \mathrm{~s}$, showing that when drying is applied only at the final step, dipping time plays a significant role in controlling film properties.

Overall, the increase in film thickness with the number of deposited bilayers clearly shows that (GN/CNC) multilayers can be built using LbL dip coating and that the growth can be adjusted by physicochemical parameters such as the ionic strength of the CNC suspension, the drying protocol, and the dipping time. The range over which the film thickness can be varied is particularly broad (variations from 220 to $1260 \mathrm{~nm}$ for example for $n=15$ ) and this exceptional behavior is not encountered with polymer/CNC multilayered systems. The ability to substantially vary the thickness most probably reflects ionic strength and drying protocolsensitive structural variations when both components are rigid nanoparticles. The influence of such parameters on porosity, roughness and nanoparticle arrangement is investigated in the next section.

\section{Structural analysis using neutron reflectivity}

Neutron reflectivity (NR) experiments were performed at the solid/air interface to complement the AFM data (Figures 2 and 3) and provide information about the particle volume fraction (and how the profile changes with distance from the substrate) as well as a precise value of the external film roughness. Figure 4 compares the NR spectra and the corresponding scattering length density (SLD) spectra for $n=7$ in the four different assembly conditions. Additionally, Supporting Information Figure S4 reports NR data and SLD profiles of (GN/CNC $10 \mathrm{mM} \mathrm{NaCl})_{n, \mathrm{I}}$ and $(\mathrm{GN} / \mathrm{CNC})_{n, \mathrm{I}}$ films for $n=2,3,4$ and 7 and of $(\mathrm{GN} / \mathrm{CNC} 10 \mathrm{mM} \mathrm{NaCl})_{n, \mathrm{~F}}$ and $(\mathrm{GN} / \mathrm{CNC})_{n, \mathrm{~F}}$ films for $n=2,4$ and 7 . 

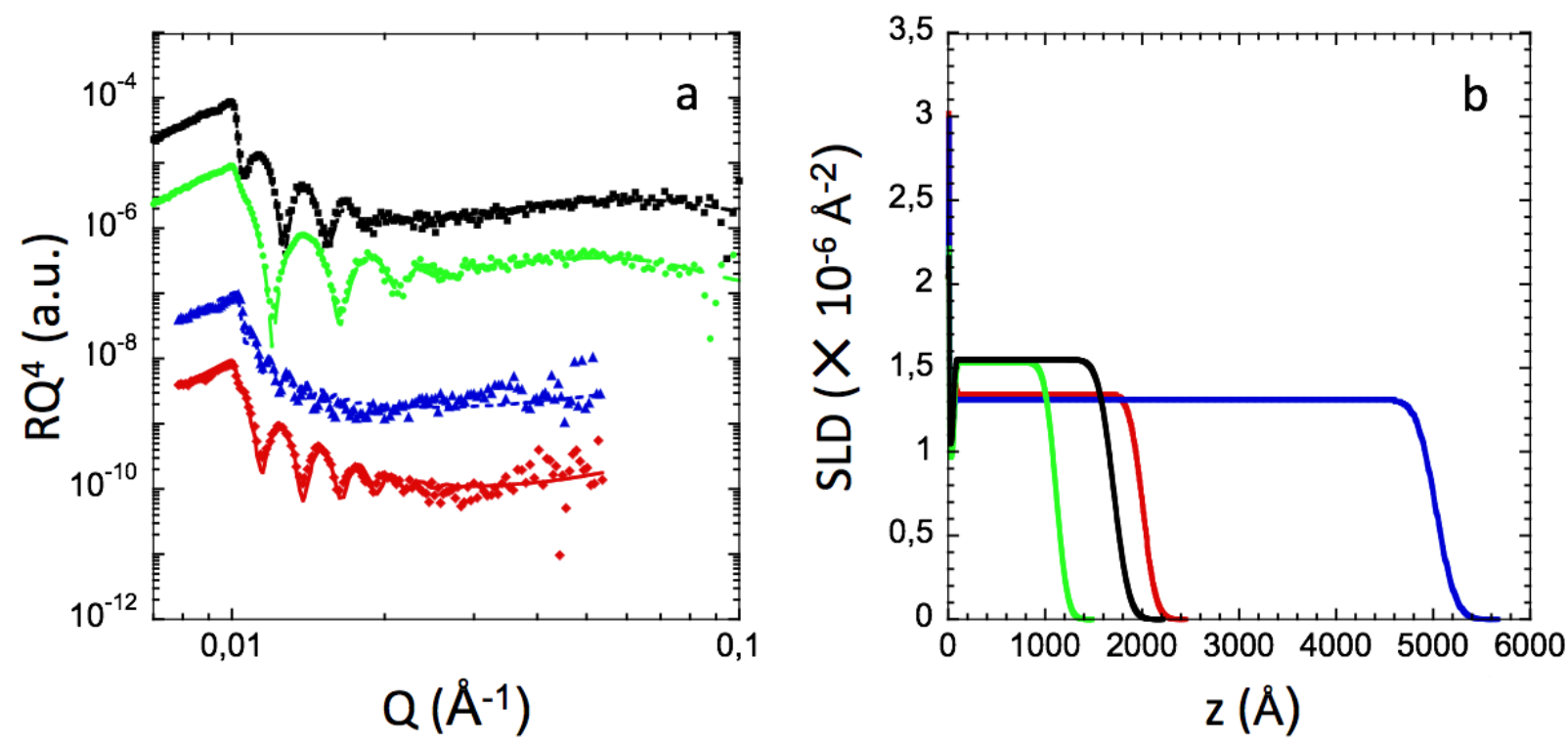

Figure 4. Neutron reflectivity spectra (a) and corresponding scattering length density profiles (b) of $(\mathrm{GN} / \mathrm{CNC})_{7, \mathrm{I}}(\diamond),(\mathrm{GN} / \mathrm{CNC})_{7, \mathrm{~F}}(\boldsymbol{\Delta}),(\mathrm{GN} / \mathrm{CNC} 10 \mathrm{mMNaCl})_{7, \mathrm{I}}(\mathbf{O})$ and $(\mathrm{GN} / \mathrm{CNC} 10$ $\mathrm{mM} \mathrm{NaCl})_{7, \mathrm{~F}}(\boldsymbol{\square})$ films. In (a), spectra were shifted for clarity and lines are best fits using the model described in the text.

An $\mathrm{RQ}^{4}$ representation was used to get rid of the substrate influence and enhance the films characteristics. All spectra in Figures 4 and Supporting Information S4 display oscillations named Kiessig fringes, whose width is related to the total film thickness. As $n$ increases, Kiessig fringes are shifted towards lower Q values and the interfringe distance decreases, revealing an increase in the total film thickness, which is consistent with aforementioned AFM film thickness data. Even if the amplitude of the oscillations rapidly diminishes with Q, due to surface roughness combined with finite resolution dampening, the presence of fringes shows that homogeneous films with well-defined interfaces were obtained in all cases. A box model was used to fit the recorded NR spectra. The model consisted in dividing the film into four layers on top of the Si substrate: a very thin layer of silicon dioxide (layer 1), the polyelectrolyte primer (layer 2), the first layer of gibbsite nanoplatelets (layer 3) and a unique layer to model the rest of the GN/CNC film (layer 4). Other models in which this last layer was divided into sublayers showed no significant increase in the fit quality. Moreover, for models where the GN/CNC layer was subdivided into multiple layers, the high roughness of the outermost layer makes the SLD values of the layers underneath unreliable as the NevotCroce perturbation breaks down. For films built using protocol F, layer 3 could be omitted. As attested by Figures 4 and Supporting Information Figure S4, a very good agreement between the experimental data and the fit could be obtained. Fitting parameters are reported in 
Supporting Information Tables S1, S2, S3 and S4. From these parameters, the SLD profiles shown in Figures $4 \mathrm{~b}$ and Supporting Information S4B, D, F and $\mathrm{H}$ could be extracted and thickness, roughness and particle volume fraction ( $\left.\phi_{\text {nanoparticle }}\right)$ could be calculated as described in the Experimental Section. The later results are summarized in Table 1.

Table 1. Thickness, roughness, particle volume fraction and porosity of $(\mathrm{GN} / \mathrm{CNC})_{n . \mathrm{I}}$, $(\mathrm{GN} / \mathrm{CNC})_{n . \mathrm{F}},(\mathrm{GN} / \mathrm{CNC} 10 \mathrm{mM} \mathrm{NaCl})_{n . \mathrm{I}}$ and $(\mathrm{GN} / \mathrm{CNC} 10 \mathrm{mM} \mathrm{NaCl})_{n . \mathrm{F}}$ films. The error is approximated as $2-5 \%$.

\begin{tabular}{|c|c|c|c|c|c|c|}
\hline$n$ & $\begin{array}{l}\text { Drying } \\
\text { protocol }\end{array}$ & $\begin{array}{c}\mathrm{NaCl} \text { content in } \\
\mathrm{CNC} \text { suspension } \\
(\mathrm{mM})\end{array}$ & Thickness (nm) & $\begin{array}{l}\text { Roughness } \\
\text { (nm) }\end{array}$ & $\begin{array}{l}\phi_{\text {nanoparticule }}(\% \\
\text { vol) }\end{array}$ & $\begin{array}{l}\text { Film } \\
\text { porosity }\end{array}$ \\
\hline 2 & \multirow{4}{*}{ I } & \multirow{7}{*}{0} & 26.3 & 8 & \multirow{4}{*}{70} & \multirow{4}{*}{30} \\
\hline 3 & & & 45.6 & 8 & & \\
\hline 4 & & & 73.9 & 9.5 & & \\
\hline 7 & & & 192.3 & 10.9 & & \\
\hline 2 & \multirow{3}{*}{$\mathrm{F}$} & & 30.2 & 8.8 & 86 & 14 \\
\hline 4 & & & 110.5 & 12 & 81 & 19 \\
\hline 7 & & & 501.5 & 15 & 70 & 30 \\
\hline 2 & \multirow{4}{*}{ I } & \multirow{7}{*}{10} & 32.8 & 7 & \multirow{4}{*}{81} & \multirow{4}{*}{19} \\
\hline 3 & & & 48.9 & 8.7 & & \\
\hline 4 & & & 62.3 & 8.9 & & \\
\hline 7 & & & 110 & 9.2 & & \\
\hline 2 & \multirow{3}{*}{$\mathrm{F}$} & & 38.9 & 8.4 & 89 & 11 \\
\hline 4 & & & 92.1 & 10.6 & 84 & 16 \\
\hline 7 & & & 169.3 & 12.6 & 84 & 16 \\
\hline
\end{tabular}

For all four systems, good agreement between the thickness measured by AFM and NR was found, as shown in the Supporting Information Figures S5, S6, S7 and S8. In addition to low $\chi^{2}$ values, this feature further supports a very good agreement between the model and experimental data. As shown by the different NR spectra and SLD profiles obtained for the same number of bilayers $(n=7)$ in the four different assembly conditions, it can be concluded that very different architectures are obtained. The quantitative analysis of the NR data in the different conditions and for the different numbers of bilayers provides useful information on the particle volume fraction/porosity along z. The fact that NR spectra can be fitted using a single layer for the GN/CNC slab of the film shows that the particle volume fraction is constant along the normal to the film in each case. In other words, whatever the conditions, a high degree of structural homogeneity is achieved in the $\mathrm{z}$ direction (perpendicular to the substrate). This 
feature is represented by a constant SLD for the outermost layer corresponding to the (GN/CNC) multilayer in the SLD profiles (Figure 4 and Supporting Information Figure S4) and agrees with previous optical and NR studies of polymer/CNC films. ${ }^{20-21,}{ }^{27-29}$ However, depending on the building conditions, two main situations can be distinguished. When intermediate drying steps are introduced in the dipping cycles (drying protocol I), the SLD of the GN/CNC film is constant whatever the number of deposited bilayers, $n$, showing that a regular and reproducible particle deposition occurs at each adsorption step. Using equation 2, the particle volume fraction can be calculated and is equal to 70 and 81 vol. $\%$ for $(\mathrm{GN} / \mathrm{CNC})_{n . \mathrm{I}}$ and $(\mathrm{GN} / \mathrm{CNC} 10 \mathrm{mM} \mathrm{NaCl})_{n . \mathrm{I}}$ films, respectively. These values show that less porous, i.e., denser films, are obtained in the presence of added salt. This result can be attributed to charge screening favoring denser packing of the nanoparticles.

Oppositely, when only one final drying step is applied after complete assembly of the film in an aqueous environment, i.e., for samples $(\mathrm{GN} / \mathrm{CNC})_{n . \mathrm{F}}$ and $(\mathrm{GN} / \mathrm{CNC} 10 \mathrm{mM} \mathrm{NaCl})_{n . \mathrm{F}}$, the SLD of the film decreases with $n$, which reveals an increasing porosity as the number of deposition steps is increased (Table 1). Still, a constant porosity along $\mathrm{z}$ is measured. As with protocol I, films built using protocol $\mathrm{F}$ with salt, for a given $n$, lead to denser, i.e., less porous, films.

Figure 5 shows the evolution of the roughness extracted from NR data as a function of the number of bilayers. Whatever the dipping protocol, the roughness increases with the number of bilayers. However, the presence of an intermediate drying step strongly reduces the film roughness that is even kept constant at a low value of about $9 \mathrm{~nm}$ in the case of (GN/CNC 10 $\mathrm{mM} \mathrm{NaCl})_{n, \mathrm{I}}$ films. 


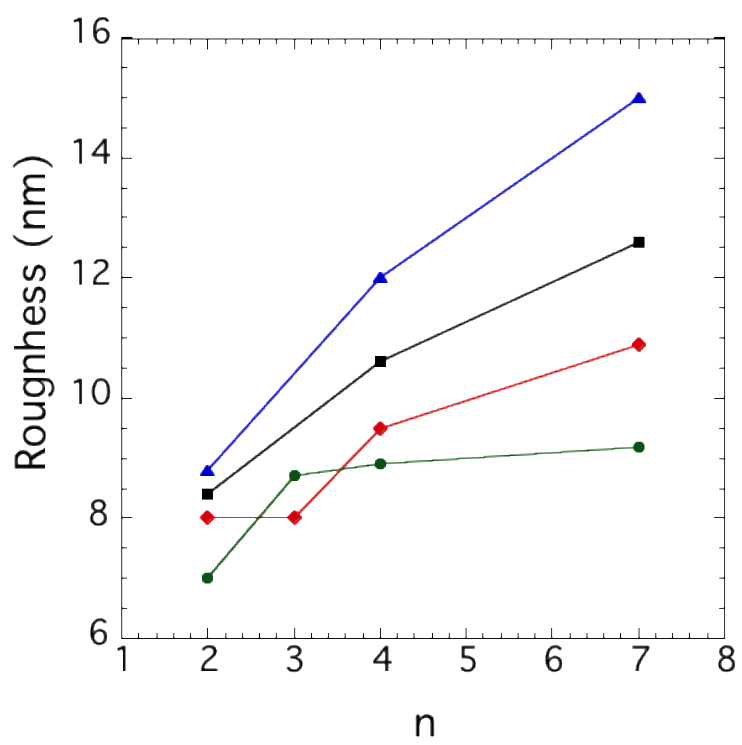

Figure 5. Film roughness extracted from NR analysis as a function of the number of deposited bilayers, $n$, for $(\mathrm{GN} / \mathrm{CNC} 10 \mathrm{mM} \mathrm{NaCl})_{n, \mathrm{I}}$ (green circles), (GN/CNC $\left.10 \mathrm{mM} \mathrm{NaCl}\right)_{n, \mathrm{~F}}$ (black squares), $(\mathrm{GN} / \mathrm{CNC})_{n, \mathrm{I}}$ (red diamonds) and (GN/CNC)$)_{n, \mathrm{~F}}$ (blue triangles).

\section{Never-dried films probed by neutron reflectivity}

To better understand the build up of films assembled using final stage drying only (protocol F), NR experiments were carried out at the solid/liquid interface on never-dried $(\mathrm{GN} / \mathrm{CNC} 10 \mathrm{mM} \mathrm{NaCl})_{n}$ films, i.e., films built in an aqueous environment without any drying steps. Such films are denoted $(\mathrm{GN} / \mathrm{CNC} 10 \mathrm{mM} \mathrm{NaCl})_{n, \mathrm{~N}}$. For these measurements, two contrasts (film $/ \mathrm{D}_{2} \mathrm{O}$ and film $/ \mathrm{H}_{2} \mathrm{O}$ ) were used to extract the hydration profile of the film in the normal direction to the surface. Results are shown in Figure 6. Unlike measurements at the solid/air interface, the spectra do not exhibit prominent Kiessig fringes. In reality, fringes are present but difficult to visualize for two reasons. The first one comes from the thickness of the films which is at the limit of the resolution of the technique and the second from the low contrast between the external environment $\left(\mathrm{D}_{2} \mathrm{O}\right.$ or $\left.\mathrm{H}_{2} \mathrm{O}\right)$ and the film, the latter being highly hydrated. 

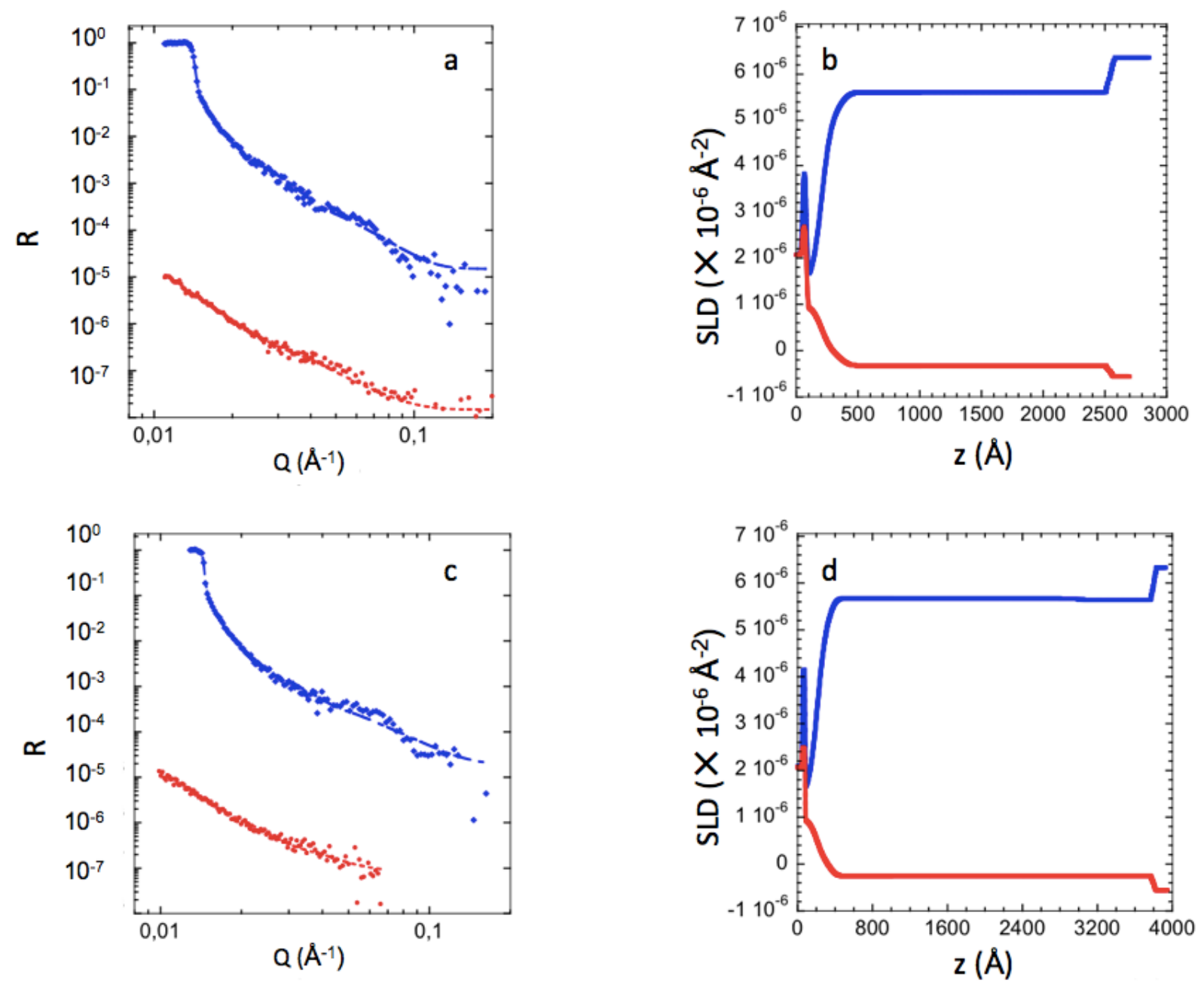

Figure 6. Neutron reflectivity spectra and corresponding scattering length density profiles for $(\mathrm{GN} / \mathrm{CNC} 10 \mathrm{mM} \mathrm{NaCl})_{n, \mathrm{~N}}$ films with $n=2$ ( $\mathrm{a}$ and $\mathrm{b}$ ) and $n=4$ (c and d). Red and blue curves correspond to measurements at the solid/ $\mathrm{D}_{2} \mathrm{O}$ and solid $/ \mathrm{H}_{2} \mathrm{O}$ interface, respectively.

The spectra in Figures $6 \mathrm{a}$ and $6 \mathrm{c}$ were fitted using the same 4-layer model as described for the solid/air case above. For each $n$ value, a single set of parameters could be used to fit both solvent conditions with good agreement, which makes it possible to unambiguously extract the SLD profiles shown in Figures $6 \mathrm{~b}$ and $6 \mathrm{~d}$ and the hydration rate of each layer constituting the film. Results are summarized in Supporting Information Table S5. For the two numbers of bilayers tested ( $n=2$ or 4$)$, the overall structure of the $(\mathrm{GN} / \mathrm{CNC})_{n, \mathrm{~N}}$ in the wet state is identical. It consists of a thin layer of approximately $15 \mathrm{~nm}$ probably corresponding to the first layer of GN with a percentage of hydration of $78 \%$, atop of which sits an extremely hydrated and very thick layer (220 nm thick and 91\% hydrated for $n=2$ and $346 \mathrm{~nm}$ thick and $87 \%$ hydrated for $n=4)$. Upon drying, these (GN/CNC $10 \mathrm{mM} \mathrm{NaCl})_{2}$ and 4,N films became samples (GN/CNC 10 $\mathrm{mM} \mathrm{NaCl})_{2}$ and 4,F, which exhibit thickness values in the dry state equal to 62 and $92 \mathrm{~nm}$, 
respectively. The drying protocol therefore resulted in a dramatic 3.5-3.7 times reduction in the thickness of the film, together with water removal.

\section{Discussion}

The results provided here clearly demonstrate the possibility to use LbL assembly to prepare hybrid all-nanoparticle GN/CNC thin films. Additionally, the growth and porosity of such films can easily be tuned by the adjustment of two experimental parameters: the presence or absence of an intermediate drying step and the ionic strength of the CNC suspension. These features are a consequence of the various interaction forces at play, whose intensity and range vary according to the physicochemical parameters of film assembly. By combining the experimental data obtained from AFM and NR with additional data from contact angle and zeta potential measurements, in this section we will attempt to shed light on the relationship between interaction forces and film structure to give a comprehensive picture of the film architecture from the four different assembly protocols.

\section{Slow growth: (GN/CNC $10 \mathrm{mM} \mathrm{NaCl})_{n, I}$ multilayer film assembly}

The build up of $(\mathrm{GN} / \mathrm{CNC} 10 \mathrm{mM} \mathrm{NaCl})_{n, \mathrm{I}}$ films starts with the adsorption of a layer of GN on the polyelectrolyte primer. This PSS-terminated sub-layer has a high charge density which favors dense adsorption of GNs by electrostatic interactions. As shown by AFM, the platelets in this first layer were adsorbed primarily flat. This geometry is favored by the low roughness of the polyelectrolyte primer. Figure 3 shows that the growth of the films (GN/CNC $10 \mathrm{mM} \mathrm{NaCl})_{n, \mathrm{I}}$ proceeds linearly from this first dense GN layer with $14 \mathrm{~nm}$ per bilayer increments. Considering the dimensions of each of the nanoparticles, namely $\sim 4 \mathrm{~nm}$ for the thickness of the GNs and $\sim 7 \mathrm{~nm}$ for the height of the cotton CNCs, it is believed that each deposited bilayer corresponds to the adsorption of a single layer of nanoplatelets and a single layer of nanorods. This situation is reminiscent of the case of (CNC/xyloglucan) multilayered films, built via non-electrostatic interactions, for which NR data revealed an internal structure consisting of a monolayer of CNCs $(7 \mathrm{~nm})$ covered by a thin layer of polymer $(2 \mathrm{~nm}) .{ }^{29}$ The presence of monolayers may however seem surprising for $\mathrm{GN} / \mathrm{CNC}$ films when considering the charge density of each of the nanoparticles. Indeed, GNs possess 5 positive charges per $\mathrm{nm}^{2}$, while CNCs have a charge density equal to 0.5 negative charges per $\mathrm{nm}^{2}$ and multiple layers of CNCs would be needed to compensate for the high GN charge density. However, beyond charge density, it is also necessary to consider the geometry and the dimensions of the objects, 
as observed by Moreau et al. in the case of PAH/CNC multilayered films. ${ }^{22}$ In their work, monolayers or dual layers of CNCs were obtained, depending on the polymer conformation. The adsorption of rigid parallelepipedic CNCs onto a GN layer can be considered to occur mostly through interactions between the charges located on one side of the nanocrystal, leaving negative charges on the opposite upper side available for interactions with the next positive GN layer. It can thus be considered that a charge reversal can be obtained even if an overall nonstoichiometric charge ratio is reached.

Surface zeta potential measurements were performed on $(\mathrm{GN} / \mathrm{CNC} 10 \mathrm{mM} \mathrm{NaCl})_{n, \mathrm{I}}$ films for $n>6$ to ensure that the polyelectrolyte primer was not influencing the values. Results in Figure 7 show a clear charge reversal between the CNC layers (integer values of $n$ ), whose zeta potential oscillates between -40 and $-66 \mathrm{mV}$, and the GN layers (half-integer values of $n$ ), which have a relatively constant zeta potential of $+20 \mathrm{mV}$.

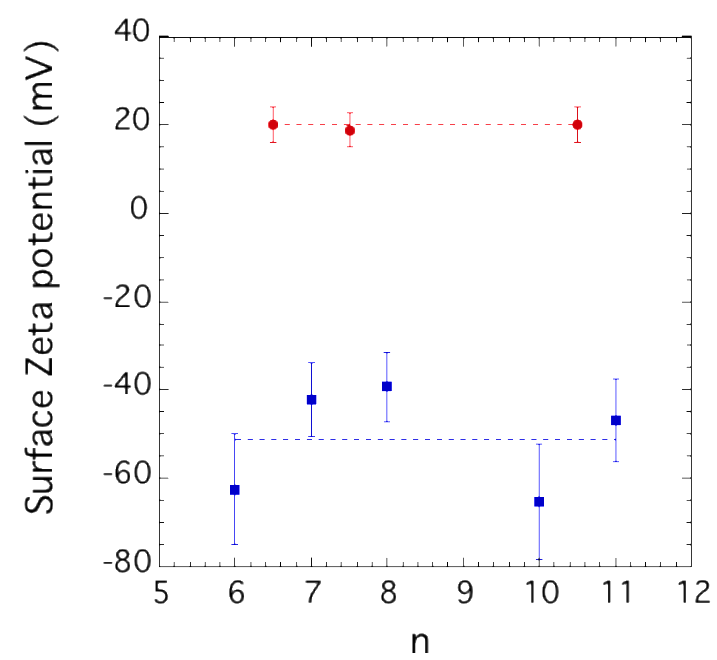

Figure 7. Surface zeta potential of (GN/CNC $10 \mathrm{mM} \mathrm{NaCl})_{n, \mathrm{I}}$ films for different $n$ values. Halfinteger $n$ values (red circles) correspond to GN-terminated films, while integer $n$ values (blue squares) correspond to CNC-terminated films.

These data first confirm that despite the low charge density of the CNCs and the fact that they are most probably deposited only in single layers, there is indeed a charge reversal each time a new layer is deposited. This situation should allow for infinite film growth. Secondly, these results might suggest that there is a separation between the CNC and the GN layers, i.e., that the film structure is not mixed but rather stratified. A systematic change in the contact angle of water droplets deposited on the external surface of the film was observed between the CNC-terminated $\left(\theta \sim 15^{\circ}\right)$ and GN-terminated $\left(\theta \sim 22^{\circ}\right)$ films (Supporting Information Figure S9). This result contrasts with the situation of $(\mathrm{GN} / \mathrm{CNC})_{n, \mathrm{~F}}$ films, which 
shows a fairly constant contact angle, irrespective of the nature of the last layer deposited. However, it would be hazardous to consider the result for (GN/CNC $10 \mathrm{mM} \mathrm{NaCl})_{n, \mathrm{I}}$ films as a proof of stratification since the contact angle depends on many parameters, including roughness and charge density. Such a regular stacking could have led to Bragg peaks in the NR spectra, as was observed in the case of PAH/deuterated CNC stratified multilayered thin films. ${ }^{21,29}$ However, here, unlike the PAH/deuterated CNC case, there is only a small difference between the SLD of the protonated CNCs used and that of the GNs and conditions for Bragg peak observation can not be met, even if the film would consist of repeating well-defined units.

Zeta potential (Figure 7) and contact angle (Supporting Information Figure S9) measurements exhibit good reproducibility with increasing number of bilayers. The same reproducibility was found for measurements of thickness increment $(14 \mathrm{~nm})$, porosity $(19 \%)$ and external roughness $(9 \mathrm{~nm}$ ) (Figure 3a, Supporting Information Figure S4F, Figure 5 and Table 1, respectively). Together, these data show that the film build up for (GN/CNC $10 \mathrm{mM}$ $\mathrm{NaCl})_{n, \mathrm{I}}$ can be repeated identically. Such robust building and possibly stratified final architecture may be attributed to the combination of electrostatic interaction forces and hydrogen-bonding. Indeed, in addition to complexation between oppositely-charged nanoparticles and counterion release, film drying after each layer deposition step favors both intra- (GN-GN or CNC-CNC) and inter-layer (GN-CNC) hydrogen-bonding when water is removed.

These data allowed us to suggest the architecture schematically depicted in Figure 8a for $(\mathrm{GN} / \mathrm{CNC} 10 \mathrm{mM} \mathrm{NaCl})_{n, \text { II }}$ It consists in repeated bilayers formed by dense GN monolayers fully covered by dense CNC monolayers, forming smooth films of low porosity and minimized thickness.

\section{Rapid growth towards hybrid porous films: $(\mathrm{GN} / \mathrm{CNC})_{n}, \mathrm{~F}$ multilayer film assembly}

The $(\mathrm{GN} / \mathrm{CNC})_{n, \mathrm{~F}}$ films, built without any added salt and no intermediate drying, constitute the other extreme situation among the four assembly protocols considered. The thickness increment of $100 \mathrm{~nm}$ per bilayer measured for $n>5$ for these films is particularly high and well above the increments observed in polymer/CNC LbL films. ${ }^{15}$ This value is much closer to the length of CNCs or the diameter of GNs than to the thickness of these nanoparticles. To explain this behavior, our hypothesis is that during construction in aqueous media, the platelets come to be adsorbed not flat but with a pronounced inclination with respect to the flat substrate. This hypothesis is supported by the fact that at the $\mathrm{pH}$ used, the positive charges are located not only on the faces but also on the edges of the platelets. As long as drying is not applied, and 
due to steric hindrance of many platelets quickly adsorbed edge-on, an orientation of the GNs parallel to the substrate appears not to be favored. In the subsequent deposition step, CNCs likely adsorb on both the faces and edges of the platelets in a disordered manner, leading to adsorbed rods oriented in all directions, including normal to the substrate surface. This non-flat adsorption propagates as further layers are deposited. Before the drying step, such a build up mechanism would lead to thick and highly hydrated mixed GN/CNC structures, which is supported by NR measurements of the solid/liquid interface on the never-dried films (Figure $6)$.

It can thus be imagined that never-dried $(\mathrm{GN} / \mathrm{CNC})_{n}$ films consist of a mixed "house of cards-like" GN/CNC network structure. The thickness of the non-dried films is about four times greater than that of the dried films. The application of drying after assembly in water causes a collapse of this swollen structure under the effect of capillary forces. The final thickness therefore represents only 13 to $24 \%$ of the thickness of the wet film, but the state of disorder is expected to be retained in the internal structure of the films, accounting for the very large thickness increment per bilayer measured. As shown in the Supporting Information Figure S3, the growth of $(\mathrm{GN} / \mathrm{CNC})_{n, \mathrm{~F}}$ films is dipping time dependent, suggesting a contribution of particle diffusion to the build up mechanism. The longer the dipping time, the more particle diffusion and rearrangement are facilitated, and the more nanoparticles can be incorporated into the wet film.

Contact angle measurements tend to support a disorganized and non-planar internal film architecture. As shown in the Supporting Information Figure S9, unlike the case of (GN/CNC $10 \mathrm{mM} \mathrm{NaCl})_{n, \mathrm{I}}$ films with intermediate drying steps, there is no change in the contact angle between CNC-terminated and GN-terminated films for $(\mathrm{GN} / \mathrm{CNC})_{n, \mathrm{~F}}$ films. The outer nanoparticle layer seems therefore to be indistinguishable by surface energy measurements, which would be consistent with a mixed, unstratified, and disordered film structure.

$\mathrm{NR}$ measurements on $(\mathrm{GN} / \mathrm{CNC})_{n, \mathrm{~F}}$ samples indicate that the porosity of the films is constant along the z-axis but increases as $n$ increases (Table I and Supporting Information Figure S4D). For $n=7$, a porosity of $30 \%$ was determined which is significantly greater than that of $19 \%$ for $(\mathrm{GN} / \mathrm{CNC} 10 \mathrm{mM} \mathrm{NaCl})_{7, \mathrm{I}}$ films. For $(\mathrm{GN} / \mathrm{CNC})_{n, \mathrm{~F}}$ films, no salt was added to the CNC suspension and the lack of charge screening favors the formation of a "loose" GN/CNC network, generating more porous structures. The probably unstratified structure of the film and the $n$-dependent porosity accounts for the higher roughness values shown in Figure 5. 
This combination of data allows us to tentatively describe $(\mathrm{GN} / \mathrm{CNC})_{n, \mathrm{~F}}$ films as extremely thick, hybrid films comprised of a porous GN/CNC structure as drawn schematically in Figure 8d. Film build up in water is likely dominated by electrostatic interactions, while drying at the end of the process makes it possible to create H-bonds between the nanoparticles only at the final step. The cohesion within the film is probably much less strong than in the preceding case because the densities are lower and the contact surface area between nanoparticles is much smaller.

\section{Intermediate situations: $(\mathrm{GN} / \mathrm{CNC} 10 \mathrm{mM} \mathrm{NaCl})_{n, \mathrm{~F}}$ and $(\mathrm{GN} / \mathrm{CNC})_{n, \mathrm{I}}$ multilayer film assembly}

$\mathrm{AFM}$ and $\mathrm{NR}$ data reveal that $(\mathrm{GN} / \mathrm{CNC} 10 \mathrm{mM} \mathrm{NaCl})_{n, \mathrm{~F}}$ films display a thickness increment per bilayer equal to $23 \mathrm{~nm}$ and low porosity around $16 \%$. While the increase in ionic strength favors higher density films and strong GN-CNC interactions, film build up in an aqueous environment without drying favors disorder and lower density films. To account for the film's structural characteristics and consider these opposing factors, we suggest that $(\mathrm{GN} / \mathrm{CNC} 10 \mathrm{mM} \mathrm{NaCl})_{n, \mathrm{~F}}$ films are composed of disordered or tilted monolayers of GNs and CNCs. A small inclination of the particles with respect to the plane of the substrate may in fact be sufficient to increase the increment from 14 to $23 \mathrm{~nm}$. This hypothesis is consistent with capping layer-independent contact angle measurements (Supporting Information Figure S9) and with roughness measurements showing higher values than in the $(\mathrm{GN} / \mathrm{CNC} 10 \mathrm{mM} \mathrm{NaCl})_{n, \mathrm{I}}$ case. The proposed structure of these films is schematically depicted in Figure $8 \mathrm{~b}$.

For $(\mathrm{GN} / \mathrm{CNC})_{n}$, f films, an increment of $48 \mathrm{~nm}$ per bilayer was measured by AFM and NR for $n>5$. Additional AFM analyses were carried out after each new deposition up to 5 bilayers. Results in Supporting Information Figure S10 clearly show a staircase-like growth with a low thickness increase of about $3.5 \mathrm{~nm}$ after adsorption of GNs and an average much larger thickness increase of $19 \mathrm{~nm}$ after adsorption of CNCs. Such a profile is consistent with the adsorption of single layers of GNs and multiple layers of CNCs. Examples of dual layers of CNCs have already been reported in the literature when the cellulose nanoparticles were associated with polycations such as $\mathrm{PAH}^{21-22}$ or $\mathrm{PEI}{ }^{46}$ due to favorable polymer conformation/flexibility and to counterions release. In our case, the combination of low ionic strength, i.e., large electric double layer, and water removal through drying after each deposition may promote attractive interactions leading to the adsorption of double or triple layers of CNCs, which could be stabilized by H-bonds and favoured by the entropic gain associated with counterions release. NR results show that these films display a relatively high 
$n$-independent porosity of $30 \%$ compared to the other films prepared. The proposed architecture of $(\mathrm{GN} / \mathrm{CNC})_{n, \text { I films is schematically represented in Figure } 8 \mathrm{c} .}$

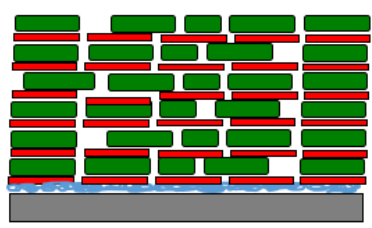

a

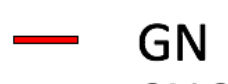

CNC

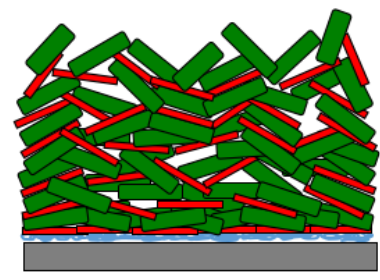

b

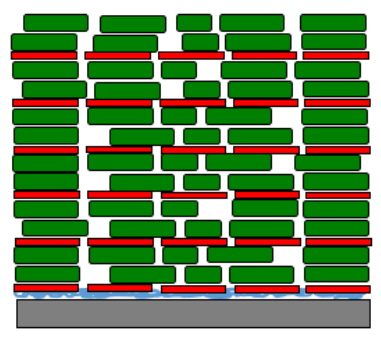

C

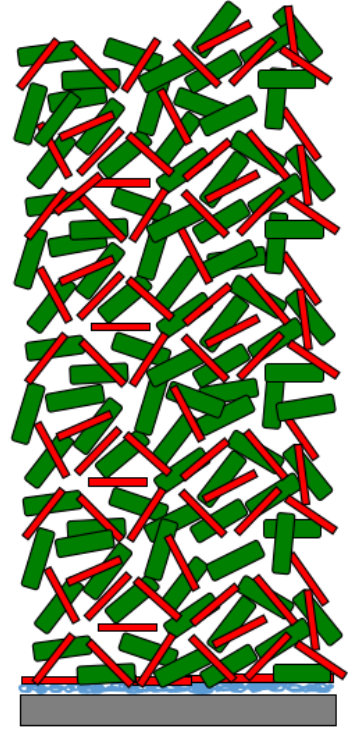

d

Figure 8. Variations in the architecture of 6-bilayered GN/CNC hybrid films built under different physicochemical conditions, namely (GN/CNC $10 \mathrm{mM})_{6, \mathrm{I}}(\mathrm{a}),(\mathrm{GN} / \mathrm{CNC} 10 \mathrm{mM})_{6, \mathrm{~F}}$ (b), $(\mathrm{GN} / \mathrm{CNC})_{6, \mathrm{I}}(\mathrm{c})$ and $(\mathrm{GN} / \mathrm{CNC})_{6, \mathrm{~F}}(\mathrm{~d})$. Stratification in a and $\mathrm{c}$ is probable and consistent with data but could not be unambiguously proven by NR due to intrinsic very small SLD difference between CNCs and GNs.

\section{Effect of salt addition}

According to the different data sets, the influence of $\mathrm{NaCl}$ can be summarized as follows: an increase in the ionic strength of the $\mathrm{CNC}$ suspension promotes the adsorption of dense single layers of CNCs, as observed for $(\mathrm{GN} / \mathrm{CNC} 10 \mathrm{mM})_{n, \mathrm{I}}$ and $(\mathrm{GN} / \mathrm{CNC} 10 \mathrm{mM})_{n, \mathrm{~F}}$ films (Figure 8a and b). The high density is attributed to charge screening and a decrease in the Debye length favoring shorter CNC-CNC and GN-CNC spacings within and between the layers. This result is reminiscent of the important effect of ionic strength and surface charge density on the thickness, roughness and porosity in nanoparticle/polyelectrolyte and allnanoparticles LbL films. ${ }^{47-49}$ Specifically, the observed densification of the CNC layers in the hybrid films with the addition of a small amount of monovalent salt in the CNC suspension is in agreement with recent work by Dammak et al. ${ }^{28}$ Their work reports a decrease in the porosity of CNC/xyloglucan multilayered films upon addition of $5 \mathrm{mM} \mathrm{NaCl}$ to the suspension of CNCs. Even though their films were not assembled by electrostatic interactions, the salt screening allows CNCs to pack more closely on the surface, as observed in this work. The addition of small amounts of salt in CNC dispersions has moreover already been shown to influence many 
properties such as particle aggregation, ${ }^{50}$ emulsion stabilization, ${ }^{51}$ the formation and organization of liquid crystal phases ${ }^{52-53}$ and the colloidal behavior of CNCs. ${ }^{54}$

Finally, data in the Supporting Information Figure $\mathrm{S} 1$ show that $1 \mathrm{mM} \mathrm{NaCl}$ is enough to cause screening and that any further addition within the limit of $10 \mathrm{mM}$ does not entail any change in film growth or volume fraction. Salt addition overall promotes a linear and slow growth, as observed for both $(\mathrm{GN} / \mathrm{CNC} 10 \mathrm{mM})_{n, \mathrm{I}}$ and $(\mathrm{GN} / \mathrm{CNC} 10 \mathrm{mM})_{n, \mathrm{~F}}$ films.

\section{Influence of the drying protocol}

As far as the drying protocol is concerned, it can be concluded that the use of drying after each layer deposition favors:

(i) homogeneous film growth with a constant volume fraction normal to the film, which is independent of the number of deposited bilayers,

(ii) a low surface roughness,

Films with enhanced homogeneity due to drying steps after each layer deposition have already been reported in the literature for $\mathrm{CNC} / \mathrm{PAH}$ multilayered films and similar rules are found to apply here to all-nanoparticle films. ${ }^{22}$ Moreau et al. state that during the drying steps, the water molecules are ejected from the film generating denser films which are further reinforced by non-electrostatic interactions such as hydrogen bonding. It is expected that such a hydrogen bonding network also develops in this case of hybrid GN/CNC LbL films. Conversely, drying at the end of the process after full assembly in water (protocol F) generates mixed GN/CNC films of higher roughness and thickness-dependent porosity, following the collapse of highly swollen structures. This drying method is able to yield very thick films with fewer deposited layers.

\section{Conclusion}

While various examples of $\mathrm{CNC} /$ polymer LbL films are reported in the literature, the building of hybrid "nanoparticle only" multilayered films associating CNCs and gibbsite nanoplatelets is demonstrated here. The influence of two parameters on the growth and structure of the films, namely the ionic strength of the $\mathrm{CNC}$ suspension $(0$ or $10 \mathrm{mM}$ added $\mathrm{NaCl})$ and the drying protocol (with drying or without drying after the deposition of each new layer) was investigated. Beyond the proof of principle of the successful preparation of homogeneous films under all four conditions probed, AFM data further show that four very different film build up behaviors are obtained, with thickness increments per bilayer ranging from 14 to $100 \mathrm{~nm}$. 
Detailed structural information (thickness, roughness, and porosity) for these thin film composites was obtained by exploiting AFM and neutron reflectivity. Depending on the conditions, thin and dense multilayered films or very thick, more porous mixed slabs, as well as intermediate situations were identified, showing a degree of structural tunability that is much more pronounced than in the case of $\mathrm{CNC}$ /polymer LbL films. Interestingly, when drying was applied after each deposited layer, dipping time-independent growth and structures were obtained that allowed the dipping time to be decreased to $10 \mathrm{~s}$. This result is another unique aspect of rigid nanoparticle-based films as the ones incorporating polymers usually require longer times to reach thermodynamic equilibrium due to conformational changes and rearrangement during deposition.

Data show that the final architecture of the films results from the interplay between the dominant interactions forces (electrostatic interactions, capillary forces, hydrogen bonding) involved. The intensity and extent of these forces strongly depend on the ionic strength and drying process, which leads to highly contrasting film structures. The influence of each parameter on the structural characteristics of the film architecture was identified, e.g. an increase in ionic strength resulted in denser films, while drying after each deposition step favored homogeneity. These results provide "design rules" to finely tailor the micro and macroscopic properties of such nanoparticle-only hybrid films to meet the needs for applications as barrier coatings or flame retardancy materials.

\section{ASSOCIATED CONTENT}

\section{Supporting Information}

The Supporting Information is available free of charge on the ACS Publications website and contains data on the effect of the ionic strength of the $\mathrm{CNC}$ suspension and of the dipping time on the growth of $(\mathrm{GN} / \mathrm{CNC})$ films, NR spectra, corresponding SLD profiles and fitting parameters in the different conditions probed as well as plots comparing thickness values extracted from AFM and NR. The SI also contains fitting parameters of NR spectra recorded on never-dried films, contact angle measurements for the different types of films and AFM data for $(\mathrm{GN} / \mathrm{CNC})_{\mathrm{n}, \mathrm{I}}$ multilayers . 


\section{AUTHOR INFORMATION}

Corresponding Author

*E-mail: bruno.jean@cermav.cnrs.fr

Present Addresses

${ }^{1}$ R.B.: School of Physical Sciences, University of Kent, Canterbury. U.K.

${ }^{2}$ E.W.: Materials Physics and Application Division, MPA-11, Los Alamos National

Laboratory, Los Alamos, New Mexico 87545, United States

\section{ACKNOWLEDGMENTS}

The authors thank the ILL for awarding a studentship to CM in the framework of the ILL PhD programme and for beamtime allocation. $\mathrm{CM}$ and $\mathrm{BJ}$ acknowledge support from ICMG FR 2607 and thank Hugues Bonnet for technical assistance. CM and BJ thank Guillaume Costa and Guillaume Nonglaton (CEA LETI, Grenoble) for access to the electrokinetic analyzer and for technical assistance.

\section{References}

1. Iler, R. K. Multilayers of Colloidal Particles. J. Colloid Interface Sci. 1966, 21, 569594.

2. Decher, G. Fuzzy Nanoassemblies: Toward Layered Polymeric Multicomposites. Science 1997, 277, 1232-1237.

3. Decher, G.; Hong, J. D.; Schmitt, J. Buildup of Ultrathin Multilayer Films by a Selfassembly Process: III. Consecutively Alternating Adsorption of Anionic and Cationic Polyelectrolytes on Charged Surfaces. Thin Solid Films 1992, 210, 831-835.

4. Hoogeveen, N. G.; Stuart, M. A. C.; Fleer, G. J.; Bohmer, M. R. Formation and Stability of Multilayers of Polyelectrolytes. Langmuir 1996, 12, 3675-3681.

5. $\quad$ Ariga, K.; Yamauchi, Y.; Rydzek, G.; Ji, Q.; Yonamine, Y.; Wu, K. C. W.; Hill, J. P. Layer-by-layer Nanoarchitectonics: Invention, Innovation, and Evolution. Chem. Lett. 2014, 43, 36-68.

6. Fakhrullin, R. F.; Lvov, Y. M. "Face-Lifting” and "Make-Up" for Microorganisms: Layer-by-Layer Polyelectrolyte Nanocoating. ACS Nano 2012, 6, 4557-4564.

7. Such, G. K.; Johnston, A. P. R.; Caruso, F. Engineered Hydrogen-Bonded Polymer Multilayers: from Assembly to Biomedical Applications. Chem. Soc. Rev. 2011, 40, 19-29. 8. Volodkin, D.; Skirtach, A.; Moehwald, H. Lbl Films as Reservoirs for Bioactive Molecules. Adv. Polym. Sci. 2011, 240, 135-161. 
9. Multilayer Thin Films: Sequential Assembly of Nanocomposite Materials, $2 d$ Edition; Decher, G., Schlenoff, J. B., Eds.; Wiley-VCH Verlag GmbH \& Co.: Weinheim, 2012

10. Habibi, Y.; Lucia, L. A.; Rojas, O. J. Cellulose Nanocrystals: Chemistry, SelfAssembly, and Applications. Chem. Rev. 2010, 110, 3479-3500.

11. Klemm, D.; Kramer, F.; Moritz, S.; Lindstrom, T.; Ankerfors, M.; Gray, D.; Dorris, A. Nanocelluloses: A New Family of Nature-Based Materials. Angew. Chem., Int. Ed. 2011, 50, 5438-5466.

12. Moon, R. J.; Martini, A.; Nairn, J.; Simonsen, J.; Youngblood, J. Cellulose Nanomaterials Review: Structure, Properties And Nanocomposites. Chem. Soc. Rev. 2011, 40, 3941-3994.

13. Šturcová, A.; Davies, G. R.; Eichhorn, S. J. Elastic Modulus and Stress-Transfer Properties of Tunicate Cellulose Whiskers. Biomacromolecules 2005, 6, 1055-1061.

14. Saito, T.; Kuramae, R.; Wohlert, J.; Berglund, L. A.; Isogai, A. An Ultrastrong Nanofibrillar Biomaterial: The Strength of Single Cellulose Nanofibrils Revealed via Sonication-Induced Fragmentation. Biomacromolecules 2013, 14, 248-253.

15. Martin, C.; Jean, B. Nanocellulose/Polymer Multilayered Thin Films: Tunable Architectures towards Tailored Physical Properties. Nord. Pulp Pap. Res. J. 2014, 29, 19-30. 16. Podsiadlo, P.; Choi, S.-Y.; Shim, B.; Lee, J.; Cuddihy, M.; Kotov, N. A. Molecularly Engineered Nanocomposites: Layer-by-Layer Assembly of Cellulose Nanocrystals.

Biomacromolecules 2005, 6, 2914-2918.

17. Podsiadlo, P.; Sui, L.; Elkasabi, Y.; Burgardt, P.; Lee, J.; Miryala, A.; Kusumaatmaja, W.; Carman, M. R.; Shtein, M.; Kieffer, J.; Lahann, J.; Kotov, N. A. Layer-by-Layer Assembled Films of Cellulose Nanowires with Antireflective Properties. Langmuir 2007, 23, 7901-7906.

18. Cranston, E. D.; Gray, D. G. Formation of Cellulose-Based Electrostatic Layer-ByLayer Films in a Magnetic Field. Sci. Technol. Adv. Mater. 2006, 7, 319-321.

19. Cranston, E. D.; Gray, D. G. Morphological and Optical Characterization of Polyelectrolyte Multilayers Incorporating Nanocrystalline Cellulose. Biomacromolecules 2006, 7, 2522-2530.

20. Cranston, E. D.; Gray, D. G. Birefringence in Spin-Coated Films Containing Cellulose Nanocrystals. Colloids Surf., A 2008, 325, 44-51.

21. Jean, B.; Dubreuil, F.; Heux, L.; Cousin, F. Structural Details of Cellulose Nanocrystals/Polyelectrolytes Multilayers Probed by Neutron Reflectivity and AFM. Langmuir 2008, 24, 3452-3458.

22. Moreau, C.; Beury, N.; Delorme, N.; Cathala, B. Tuning the Architecture of Cellulose Nanocrystal-Poly(allylamine hydrochloride) Multilayered Thin Films: Influence of Dipping Parameters. Langmuir 2012, 28, 10425-10436.

23. de Mesquita, J. P.; Donnici, C. L.; Pereira, F. V. Biobased Nanocomposites from Layer-by-Layer Assembly of Cellulose Nanowhiskers with Chitosan. Biomacromolecules 2010, 11, 473-480.

24. de Mesquita, J. P.; Patricio, P. S.; Donnici, C. L.; Petri, D. F. S.; de, O. L. C. A.; Pereira, F. V. Hybrid Layer-By-Layer Assembly Based on Animal and Vegetable Structural Materials: Multilayered Films of Collagen and Cellulose Nanowhiskers. Soft Matter 2011, 7, 4405-4413.

25. Cerclier, C.; Cousin, F.; Bizot, H.; Moreau, C.; Cathala, B. Elaboration of Spin-Coated Cellulose-Xyloglucan Multilayered Thin Films. Langmuir 2010, 26, 17248-17255.

26. Cerclier, C.; Moreau, C.; Guyomard-Lack, A.; Bonnin, E.; Bizot, H.; Cathala, B. Semi Reflective Biopolymer Layers for the Detection of Biomass Hydrolytic Enzymatic Activities. MRS Online Proc. Libr. 2011, 1326 
27. Cerclier, C. V.; Guyomard-Lack, A.; Cousin, F.; Jean, B.; Bonnin, E.; Cathala, B.; Moreau, C. Xyloglucan-Cellulose Nanocrystal Multilayered Films: Effect of Film Architecture on Enzymatic Hydrolysis. Biomacromolecules 2013, 14, 3599-3609. 28. Dammak, A.; Moreau, C.; Azzam, F.; Jean, B.; Cousin, F.; Cathala, B. Influence of Cellulose Nanocrystals Concentration and Ionic Strength on the Elaboration of Cellulose Nanocrystals-Xyloglucan Multilayered Thin Films. J. Colloid Interface Sci. 2015, 460, $214-$ 220.

29. Jean, B.; Heux, L.; Dubreuil, F.; Chambat, G.; Cousin, F. Non-Electrostatic Building of Biomimetic Cellulose-Xyloglucan Multilayers. Langmuir 2009, 25, 3920-3923.

30. Aulin, C.; Salazar-Alvarez, G.; Lindstrom, T. High Strength, Flexible and Transparent Nanofibrillated Cellulose-Nanoclay Biohybrid Films with Tunable Oxygen and Water Vapor Permeability. Nanoscale 2012, 4, 6622-6628.

31. Gabr, M. H.; Phong, N. T.; Abdelkareem, M. A.; Okubo, K.; Uzawa, K.; Kimpara, I.; Fujii, T. Mechanical, Thermal, and Moisture Absorption Properties of Nano-Clay Reinforced Nano-Cellulose Biocomposites. Cellulose 2013, 20, 819-826.

32. Wang, J.; Cheng, Q.; Lin, L.; Jiang, L. Synergistic Toughening of Bioinspired Poly(vinyl alcohol)-Clay-Nanofibrillar Cellulose Artificial Nacre. ACS Nano 2014, 8, 27392745.

33. Wu, C.-N.; Saito, T.; Yang, Q.; Fukuzumi, H.; Isogai, A. Increase in the Water Contact Angle of Composite Film Surfaces Caused by the Assembly of Hydrophilic Nanocellulose Fibrils and Nanoclay Platelets. ACS Appl. Mater. Interfaces 2014, 6, 12707 12712.

34. Tang, Z.; Kotov, N. A.; Magonov, S.; Ozturk, B. Nanostructured Artificial Nacre. Nat. Mater. 2003, 2, 413-418.

35. van der Beek, D.; Lekkerkerker, H. N. W. Liquid Crystal Phases of Charged Colloidal Platelets. Langmuir 2004, 20, 8582-8586.

36. Wijnhoven, J. E. G. J.; van't Zand, D. D.; van der Beek, D.; Lekkerkerker, H. N. W. Sedimentation and Phase Transitions of Colloidal Gibbsite Platelets. Langmuir 2005, 21, 10422-10427.

37. Wierenga, A. M.; Lenstra, T. A. J.; Philipse, A. P. Aqueous Dispersions of Colloidal Gibbsite Platelets: Synthesis, Characterisation and Intrinsic Viscosity Measurements. Colloids Surf., A 1998, 134, 359-371.

38. Olivier, C.; Moreau, C.; Bertoncini, P.; Bizot, H.; Chauvet, O.; Cathala, B. Cellulose Nanocrystal-Assisted Dispersion of Luminescent Single-Walled Carbon Nanotubes for Layerby-Layer Assembled Hybrid Thin Films. Langmuir 2012, 28, 12463-12471.

39. Trigueiro, J. P. C.; Silva, G. G.; Pereira, F. V.; Lavall, R. L. Layer-by-Layer Assembled Films of Multi-Walled Carbon Nanotubes with Chitosan and Cellulose Nanocrystals. J. Colloid Interface Sci. 2014, 432, 214-220.

40. Revol, J. F.; Bradford, H.; Giasson, J.; Marchessault, R. H.; Gray, D. G. Helicoidal Self-Ordering of Cellulose Microfibrils in Aqueous Suspension. Int. J. Biol. Macromol. 1992, $14,170-172$.

41. Azzam, F.; Heux, L.; Putaux, J.-L.; Jean, B. Preparation By Grafting Onto, Characterization, and Properties of Thermally Responsive Polymer-Decorated Cellulose Nanocrystals. Biomacromolecules 2010, 11, 3652-3659.

42. Elazzouzi-Hafraoui, S.; Nishiyama, Y.; Putaux, J.-L.; Heux, L.; Dubreuil, F.; Rochas, C. The Shape and Size Distribution of Crystalline Nanoparticles Prepared by Acid Hydrolysis of Native Cellulose. Biomacromolecules 2008, 9, 57-65.

43. Campbell, R. A.; Wacklin, H. P.; Sutton, I.; Cubitt, R.; Fragneto, G. FIGARO: the New Horizontal Neutron Reflectometer at the ILL. Eur. Phys. J. Plus 2011, 126, 107. 
44. Wang, H.; Qian, C.; Roman, M. Effects of pH and Salt Concentration on the Formation and Properties of Chitosan-Cellulose Nanocrystal Polyelectrolyte-Macroion Complexes. Biomacromolecules 2011, 12, 3708-3714.

45. Izquierdo, A.; Ono, S. S.; Voegel, J. C.; Schaaf, P.; Decher, G. Dipping versus Spraying: Exploring the Deposition Conditions for Speeding Up Layer-by-Layer Assembly. Langmuir 2005, 21, 7558-7567.

46. Kan, K. H. M. C., E. D. Mechanical Testing of Thin Film Nanocellulose Composites Using Buckling Mechanics. TAPPI J. 2013, 12 (4), 9-17.

47. Lee, D.; Gemici, Z.; Rubner, M. F.; Cohen, R. E. Multilayers of oppositely charged $\mathrm{SiO} 2$ nanoparticles: effect of surface charge on multilayer assembly. Langmuir 2007, 23, 8833-8837.

48. Lee, S. W.; Kim, B.-S.; Chen, S.; Shao-Horn, Y.; Hammond, P. T. Layer-by-layer assembly of all carbon nanotube ultrathin films for electrochemical applications. J. Am. Chem. Soc. 2008, 131, 671-679.

49. Ostrander, J. W.; Mamedov, A. A.; Kotov, N. A. Two Modes of Linear Layer-byLayer Growth of Nanoparticle-Polylectrolyte Multilayers and Different Interactions in the Layer-by-layer Deposition. J. Am. Chem. Soc. 2001, 123, 1101-1110.

50. Cherhal, F.; Cousin, F.; Capron, I. Influence of Charge Density and Ionic Strength on the Aggregation Process of Cellulose Nanocrystals in Aqueous Suspension, as Revealed by Small-Angle Neutron Scattering. Langmuir 2015, 31, 5596-5602.

51. Kalashnikova, I.; Bizot, H.; Cathala, B.; Capron, I. Modulation of Cellulose Nanocrystals Amphiphilic Properties to Stabilize Oil/Water Interface. Biomacromolecules 2011, 13, 267-275.

52. Hirai, A.; Inui, O.; Horii, F.; Tsuji, M. Phase Separation Behavior in Aqueous Suspensions of Bacterial Cellulose Nanocrystals Prepared by Sulfuric Acid Treatment. Langmuir 2009, 25, 497-502.

53. Araki, J.; Kuga, S. Effect of Trace Electrolyte on Liquid Crystal Type of Cellulose Microcrystals. Langmuir 2001, 17, 4493-4496.

54. Boluk, Y.; Lahiji, R.; Zhao, L.; McDermott, M. T. Suspension viscosities and shape parameter of cellulose nanocrystals (CNC). Colloids Surf., A 2011, 377, 297-303. 
TABLE OF CONTENTS GRAPHICS

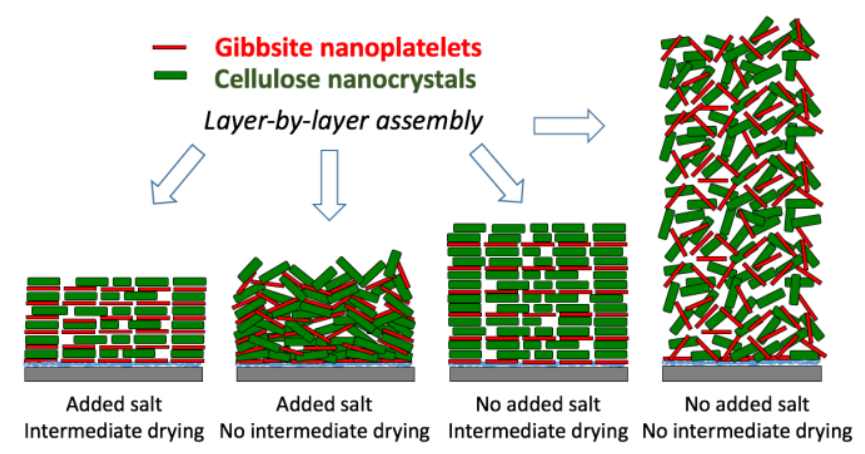

